\title{
ON THE DECOMPOSITION OF UNITARY REPRESENTATIONS OF LIE GROUPS
}

\author{
F. I. MAUTNER
}

1. Let $G$ be a connected Lie group and

$$
g \rightarrow U(g)
$$

a continuous ${ }^{1}$ unitary representation of $G$ in a separable Hilbert space $\mathfrak{S}$ over the complex numbers. Let $a$ be any element of the Lie algebra $\$ 5$ of $G$; then exp $\theta a$, as defined in [1, chap. IV, $\S \mathrm{VIII}]$ is an element of the group $G$ and as $\theta$ varies over the real line, $\exp \theta a$ varies over a certain one-parameter subgroup of $G$. Therefore the operators $U(\exp \theta a)$ form a one-parameter group of unitary operators in $\mathfrak{S}$. Hence there exists, as is well known, a self-adjoint (hypermaximal) operator $A$ defined by $\lim _{\theta \rightarrow 0}(1 / \theta)(U(\exp \theta a)-I) x=i A x$ where $D_{A}$, the domain of definition of $A$, is the set of those $x \in \mathfrak{F}$ for which this limit exists. And

$$
U(\exp \theta a)=\exp i \theta A,
$$

where the exp on the right is the usual exponential of a self-adjoint operator. $D_{A}$ is a dense linear subspace of $\mathfrak{E}$; it has been proved in [2] that as $a$ varies over $\&$ the intersection of all the corresponding domains $D_{A}$ is still a dense linear subspace of $\mathfrak{S}$. It is easy to deduce from this $[5, \S 5]$ that the mapping

$$
a \rightarrow i A
$$

defines a representation of the Lie algebra $(B)$ by means of linear transformations in a certain dense linear subspace of $\mathfrak{S}$.

Now suppose we are given a generalized direct sum (direct integral) decomposition

$$
\mathfrak{S}=\int_{\oplus} \mathfrak{W}_{t}
$$

of $\mathfrak{E}$ into Hilbert or finite-dimensional spaces $\mathfrak{S}_{t}$ as defined ${ }^{2}$ in [7].

Received by the editors April 27, 1950.

${ }^{1}$ By continuous we shall mean throughout continuous in the weak (or what is in this case the same, the strong) topology for operators as defined in [6]. Numbers in brackets refer to the bibliography at the end of the paper.

2 To define a direct integral the set of indices $t$ must form a measure space. All statements about a measure on the set of points $t$ such as a.a. $t$ (almost all $t$ ) refer to this fixed measure. The only other measure which occurs is the Haar measure on $G$ and in one instance its image under the mapping (2.8). 
Suppose that the decomposition (1.4) is such that $U(g)$ is decomposable (as defined in [7, part II]) for every $g \in G$. Thus there exist for each $g$ an operator-valued function $U(g, t)$ of $t$ whose value is for each $g \in G$ and a.e. $t$ a unitary operator in $\mathfrak{E}_{t}$. Under these conditions it has been seen in $[5, \S 4]$ that there exists for each of the above operators $A$ an operator-valued function $A(t)$ where $A(t)$ is (after a possible change on a $t$-set of measure zero) for each $t$ a self-adjoint operator in the space $\mathfrak{S}_{t}$ such that

$$
U(g, t)=\exp i \theta A(t) \quad \text { for a.a. } t,
$$

where $g=\exp \theta a$. Hence if we change $U(g, t)$ for each $g$ on a suitable $t$-set of $s$-measure zero we obtain a new operator $\tilde{U}(g, t)$ such that

$$
\tilde{U}(g, t)=\tilde{U}(\exp \theta a, t)=\exp i \theta A(t) \quad \text { for all } t \text {. }
$$

We shall prove in the present note the following theorem.

THEOREM. Given any continuous unitary representation (1.1) of a connected Lie group $G$ in a separable Hilbert space $\mathfrak{S}$ and a direct integral $\mathfrak{E}=\int \oplus \mathfrak{S}_{t}$ under which the operator $U(\mathrm{~g})$ decomposes for each $g \in G$ into (say) $U(g, t)$. Then it is possible to change $U(g, t)$ for each $g$ on a $t$-set of $s$-measure zero into a unitary operator $\tilde{U}(g, t)$ in $\mathfrak{S}_{t}$ such that the mapping

$$
g \rightarrow \tilde{U}(g, t)
$$

is a continuous unitary representation of the group $G$ for every $t$.

Moreover (after a possible change of the spaces $\mathfrak{S}_{t}$ on a set of measure zero) there exists for each $t$ a dense linear subspace $\mathfrak{W}_{t}^{0}$ of $\mathfrak{W}_{t}$ such that $D_{A(t)} \supseteq \mathfrak{S}_{t}^{0}, A(t) \mathfrak{E}_{t}^{0} \subseteq \mathfrak{S}_{t}^{0}$, and if $A_{0}(t)$ denotes the restriction of $A(t)$ to the subspace $\mathfrak{S}_{i}^{0}$ then the mapping

$$
a \rightarrow A_{0}(t)
$$

is a representation of the Lie algebra $(\$)$.

We shall prove this theorem in $\$ 2$. It is an improvement of [5, Theorem 1.1] in the case of Lie groups. However, the present proof is entirely different from that of [5, Theorem 1.1]. In the present proof we shall use properties of the Lie algebra very strongly, whereas in [5] we used only properties which are true for any (separable) locally compact group, but from which we could conclude a slightly weaker result only.

Our present theorem has various applications. For instance it implies immediately a strengthening in the case of Lie groups of $[\mathbf{5}$, Theorem 2.1] on the integral representation of positive definite func- 
tions. Indeed it is clear that we can now assert that for connected Lie groups this integral representation is in terms of continuous elementary positive definite functions.

Another application is that the argument of $[5, \S 6]$ can be both shortened and generalized with the use of our present theorem. We intend to discuss this elsewhere in detail and mention now only that one of the main results of [5], namely Theorem 6.1 (on the structure of the unitary continuous representation of the Lorentz group) holds under more general conditions. It can be seen easily that with the use of the above theorem we can avoid in the argument of $[5, \S 6]$ the known fact that to any representation of the Lie algebra of the Lorentz group (satisfying certain conditions) there corresponds a unitary representation of the Lorentz group. Since this extendability appears to be unknown for arbitrary (or even semisimple) Lie groups, we have herewith removed one of the difficulties of generalizing Theorem 6.1 of $\$ 5$.

2. Proof of theorem. Let $a_{1}, a_{2}, \cdots, a_{n}$ form a base of the Lie algebra $B$ over the real numbers. Then it is well known that every element $g$ of $G$ can be written (in many ways) as a finite product

$$
\left(\exp \theta_{k_{1}} a_{k_{1}}\right)\left(\exp \theta_{k_{2}} a_{k_{2}}\right) \cdots,
$$

where $\theta_{k_{1}}, \theta_{k_{2}}, \cdots$ are real numbers. Let $G_{0}$ be the subgroup of those elements of $G$ for which at least one expression (2.1) is with $\theta_{k_{1}}$, $\theta_{k_{2}}, \cdots$ rational.

The operators $A_{1}(t), A_{2}(t), \cdots, A_{n}(t)$ which correspond to the basis elements $a_{1}, a_{2}, \cdots, a_{n}$ in the sense of equation (1.5) may of course be changed on an arbitrary $t$-set of measure zero; but we can clearly assume that for each $j=1,2, \cdots, n$ a choice has been made for $A_{j}(t)$ such that $A_{j}(t)$ is self-adjoint for all $t$.

Let us now define for $g \in G$ and for every $t$ a unitary operator $\tilde{U}(g, t)$ on $\mathfrak{S}_{t}$ by

$$
\widetilde{U}(g, t)=\exp i \theta_{k_{1}} A_{k_{1}}(t) \exp i \theta_{k_{2}} A_{k_{2}}(t) \cdots,
$$

where $g$ is given by (2.1). It has been proved in $[5, \S 4]$ that $\exp i A(t)$ depends measurably on $t$ in the sense of $[7$, Definition 4$]$ and that

$$
U(\exp a, t)=\exp i A(t)
$$

for a.a. $t$,

where $U(\exp a, t)$ is the operator-valued function of $t$ into which the unitary operator $U(\exp a)$ decomposes (for fixed $a \in(B)$ under the given direct integral (1.4). Hence (2.2) implies that $\widetilde{U}(g, t)$ depends for each $g \in G$ measurably on $t$ and 


$$
\tilde{U}(g, t)=U(g, t)
$$

for a.a.t.

Since $g \rightarrow U(g)$ is a homomorphism ${ }^{3}$

$$
\prod \exp \theta_{k} a_{k}=1=\text { identity element of } G
$$

implies

$$
\prod \exp i \theta_{k} A_{k}=I=\text { identity transformation of } \mathfrak{S} \text {. }
$$

Hence

$$
\prod \exp i \theta_{k} A_{k}(t)=I_{t}
$$

for a.a.t.

By taking the union of at most countably many exceptional $t$-sets of measure zero, it follows, since $G_{0}$ is a countable group, that (2.2) establishes a well-defined (that is, single-valued) homomorphism $g \rightarrow \tilde{U}(g, t)$ of $G_{0}$ for all $t$ outside of one set of measure zero, hence, after a trivial change, for all $t$.

Now let $g$ be an arbitrary element of $G$. We can write ${ }^{3}$

$$
g=\prod_{j=1}^{n} \exp \theta_{k_{j}} a_{k_{j}}
$$

and put

$$
g_{m}=\prod \exp \theta_{k_{j}}^{(m)} a_{k_{j}}
$$

where for each $k$ the $\theta_{k}^{(m)}$ are a sequence of rational numbers converging to $\theta_{k}$. Therefore $g_{m} \rightarrow g$ and $g_{n} \in G_{0}$. But $\theta_{k}^{(m)} \rightarrow \theta_{k}$ implies

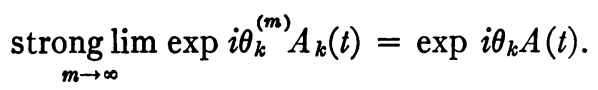

Thus strong $\lim _{m \rightarrow \infty} \tilde{U}\left(g_{m}, t\right)$ exists for all $t$. Now suppose $g_{m}^{\prime}$ is another sequence of elements of $G_{0}$ and $g_{m}^{\prime} \rightarrow g$. Then $g_{m}^{-1} g_{m}^{\prime} \rightarrow 1$. Hence we can find for $g_{m}^{-1} g_{m}^{\prime}$ an expression (2.1) such that $\lim _{m \rightarrow \infty} \theta_{k_{j}}^{(m)}=0$ for each $k$. It follows ${ }^{4}$ that $\tilde{U}\left(g_{m}^{-1} g_{m}^{\prime}, t\right)$ is the same no matter what expression (2.1) we choose for $g_{m}^{-1} g_{m}^{\prime}$. Hence

strong $\lim \tilde{U}\left(g_{m}^{-1} g_{m}^{\prime}, t\right)=I_{t} \quad$ for all $t$ outside of one fixed null set,

${ }^{3}$ Such expressions as $\prod_{k} \exp \theta_{k} a_{k}$ (or $\prod_{j} \exp \theta_{k_{j}} a_{k_{j}}$ ) stand for finite products of the group elements $\exp \theta_{k} a_{k}$ (or $\exp \theta_{k j} a_{k_{j}}$ ). Since $G$ need not be commutative we make the convention that $\prod_{i} g_{i}$ shall denote the (finite) product $g_{1} g_{2} \cdots$ in this order.

${ }^{4}$ For instance let $\psi_{1}^{m}, \cdots, \psi_{n}^{m}$ be the canonical coordinates of the second kind of $g_{m}^{\prime} g_{m}^{-1}$ for $m$ greater than some $m_{0}$ and put $U_{1}\left(g_{m}^{\prime} g_{m}^{-1}, t\right)=\exp i \theta_{1} A_{1}(t) \cdots \exp i \theta_{n} A_{n}(t)$. Since $G_{0}$ is countable we have after a change on one $t$-set of measure zero $U_{1}\left(g_{m}^{\prime} g_{m}^{-1}, t\right)$ $=\widetilde{U}\left(g_{m}^{\prime} g_{m}^{-1}, t\right)$ for all $t$ and $m>m_{0}$ and hence the desired convergence. 
therefore

$$
\text { strong } \lim \tilde{U}\left(g_{m}^{\prime}, t\right)=\text { strong } \lim \tilde{U}\left(g_{m}, t\right) \quad \text { for all } t \text {. }
$$

Therefore

$$
\widetilde{U}(g, t)=\underset{m \rightarrow \infty}{\operatorname{strong}} \lim \tilde{U}\left(g_{m}, t\right)
$$

defines for every $t$ a single-valued mapping $g \rightarrow \tilde{U}(g, t)$ of $G$ into the group of unitary operators on $\mathfrak{S}_{t}$.

Now if $h$ is another arbitrary element of $G$ and

$$
h=\prod_{k_{j}} \exp \phi_{k_{j}} a_{k_{j}}
$$

then we put

$$
h_{m}=\prod_{k_{j}} \exp \phi_{k_{j}}^{(m)} a_{k_{j}}
$$

with rational $\phi_{k_{j}}^{(m)}$ converging to $\phi_{k_{j}}$, and conclude from

$$
\tilde{U}\left(g_{m} h_{m}, t\right)=\tilde{U}\left(g_{m}, t\right) \tilde{U}\left(h_{m}, t\right) \quad \text { for all } t
$$

that

$$
\tilde{U}(g h, t)=\tilde{U}(g, t) \tilde{U}(h, t) \quad \text { for all } t ;
$$

thus $g \rightarrow \tilde{U}(g, t)$ is a well defined homomorphism of $G$ for every $t$. Note also that $\tilde{U}\left(g^{-1}, t\right)=\tilde{U}(g, t)^{*}$. For if $g=\exp \left(\theta_{1} a_{1}\right) \cdot \exp \left(\theta_{2} a_{2}\right) \cdot \ldots$ $\cdot \exp \left(\theta_{r} a_{r}\right)$, then $g^{-1}=\exp \left(-\theta_{r} a_{r}\right) \cdot \cdots \exp \left(-\theta_{2} a_{2}\right) \cdot \exp \left(-\theta_{1} a_{1}\right)$. Hence by what we have just proved

$$
\begin{aligned}
\tilde{U}\left(g^{-1}, t\right) & =\exp \left(-i \theta_{r} A_{r}(t)\right) \cdots \exp \left(-i \theta_{2} A_{2}(t)\right) \exp \left(-i \theta_{1} A_{1}(t)\right) \\
& =\tilde{U}(g, t)^{*} .
\end{aligned}
$$

We have therefore proved that the mapping $g \rightarrow \tilde{U}(g, t)$ is a unitary representation of $G$ for every $t$.

Now ${ }^{5}$ let us take any one $t$ and keep it fixed. Let $x$ and $y$ be any two elements of $\mathfrak{S}_{t}$. We wish to prove that the inner product

$$
(\tilde{U}(g, t) x, y)
$$

is a Haar-measurable function of $g$. Let $g_{0}$ be an arbitrary element of $G$ and $\Gamma$ a sufficiently small neighborhood of $g_{0}$ such that every $g \in \Gamma$ can be written uniquely as

$$
g=g_{1}\left(\theta_{1}\right) g_{2}\left(\theta_{2}\right) \cdots g_{n}\left(\theta_{n}\right) g_{0}
$$

\footnotetext{
- Added in proof. The remainder of this proof can be simplified and shortened.
} 
where the $g_{k}\left(\theta_{k}\right)$ form the one-parameter subgroup of $G$ determined by the element $a_{k}$ of the Lie algebra: $g_{k}\left(\theta_{k}\right)=\exp \theta_{k} a_{k}$. The real numbers $\theta_{k}$ are the canonical coordinates of the second kind (cf. $\left[8\right.$, p. 193]) of the element $g g_{0}^{-1}$. It is well known that if $\Gamma$ is chosen to be a sufficiently small neighborhood of $g_{0}$, then the mapping

$$
g \rightarrow\left(\theta_{1}, \theta_{2}, \cdots, \theta_{n}\right)
$$

defines a homeomorphism of $\Gamma$ to some open set $\Theta$ in Euclidean $n$ space. Under this homeomorphism there corresponds to every complex-valued function $f(g)$ on $\Gamma$ a function of the $\theta_{k}$ which we denote by $f\left(\theta_{1}, \cdots, \theta_{n}\right)$.

The restriction of the Haar-measure on $G$ to $\Gamma$ is taken by the mapping (2.8) into a certain measure defined on $\Theta$ with respect to which all continuous functions on $\Theta$ are measurable.

Now introduce in our fixed space $\mathfrak{S}_{t}$ a complete orthonormal system and with respect to it form the matrix coefficients $u_{i j}(g)$ of the operator $\tilde{U}(g, t)$. Under (2.8) the restriction of $u_{i j}(g)$ to $\Gamma$ goes over into a certain function $u_{i j}\left(\theta_{1}, \cdots, \theta_{n}\right)$. Equation (2.7) implies

$$
\tilde{U}(g, t)=\tilde{U}\left(g_{1}\left(\theta_{1}\right), t\right) \cdot \tilde{U}\left(g_{2}\left(\theta_{2}\right), t\right) \cdot \cdots \cdot \tilde{U}\left(g_{n}\left(\theta_{n}\right), t\right) \tilde{U}\left(g_{0}, t\right)
$$

for $g \in \Gamma$. Therefore for the matrix coefficients we obtain

$$
\text { (2.9) } \begin{aligned}
u_{i k}\left(\theta_{1} \cdots \theta_{n}\right) & =\sum_{j_{1}, \cdots, j_{n}} u_{i j_{1}}\left(g\left(\theta_{1}\right)\right) u_{j_{1} j_{2}}\left(g\left(\theta_{2}\right)\right) \cdots u_{j_{n-1} j_{n}}\left(g\left(\theta_{n}\right)\right) u_{j_{n}}(0, \cdots, 0) .
\end{aligned}
$$

Equation (2.2) implies that

$$
\tilde{U}\left(g\left(\theta_{k}\right), t\right)=\exp i \theta_{k} A(t) .
$$

Hence the inner product $\left(\tilde{U}\left(g\left(\theta_{k}\right), t\right) x, y\right)$ is for each fixed $t$ and $x, y \in \mathfrak{S}_{t}$ a continuous function of the one real variable $\theta_{k}$, which is a well known property of any one-parameter group of unitary operators defined by equation (2.10). Therefore the function $u_{j_{k-1} j_{k}}\left(\theta_{k}\right)$ of equation (2.9) is for each pair $j_{k-1}, j_{k}$ a continuous function. Therefore $u_{i k}\left(\theta_{1}, \cdots, \theta_{n}\right)$ is for each $i, k$ a measurable function of $\theta_{1}, \cdots, \theta_{n}$ (with respect to the above measure), being the (finite or infinite) sum (2.9) of continuous functions $u_{i j_{1}}\left(g\left(\theta_{1}\right)\right) \cdots$ $\cdot u_{j_{n-1} j_{n}}\left(g\left(\theta_{n}\right)\right) u_{j_{n} n}(0, \cdots, 0)$. Hence the restriction of $u_{i k}(g)$ to $\Gamma$ is a measurable function of $g$. The element $g_{0}$ of $G$ was arbitrary. Let us therefore choose a countable number of them such that the union of the corresponding neighborhoods $\Gamma$ is equal to $G$ (this is easily seen to be possible). Then the above implies immediately that $u_{i k}(g)$ is a Haar-measurable function on the whole of $G$. Now let 
$x_{1}, x_{2}, \cdots, y_{1}, y_{2}, \cdots$ be the expansion coefficients of $x, y\left(\in \mathfrak{W}_{t}\right)$ respectively, with respect to the above complete orthonormal system in $\mathfrak{S}_{t}$. Then

$$
(\tilde{U}(g, t) x, y)=\sum_{i, k} u_{i k}(g) x_{i} \bar{y}_{k}
$$

hence $(\tilde{U}(g, t) x, y)$ is a Haar-measurable (complex-valued) function of $g$ for every fixed $t$ and any pair of elements $x, y$ of this $\mathfrak{W}_{t}$.

Thus we have proved that $g \rightarrow \tilde{U}(g, t)$ is a "weakly" measurable unitary representation of $G$ for every $t$. Since the space $\mathfrak{S}_{t}$ is separable, this implies that $\tilde{U}(g, t)$ is continuous in $g$ for every fixed $t$, which completes the proof of the first part of our theorem.

To prove the assertion about the representations of the Lie algebra (\$), we use the results of $[5, \S \S 4$ and 5]: To the self-adjoint operator $A$ defined by equation (1.2) above corresponds in accordance with [5, $\S 4$ ] an operator-valued function $A(t)$ such that $A(t)$ is a self-adjoint operator whose domain $D_{A(t)}$ is a dense subspace of $\mathfrak{W}_{t}$. Moreover it has been shown in $[5, \S 4]$ (cf. [5, Lemma 4.4]) that equation (1.5) above is true and hence after a change on a set of measure zero even equation (1.6) is true. Hence we can apply the results of $[5, \S 5] \mathrm{im}$ mediately and conclude that there exists a certain dense linear subspace $\mathfrak{W}_{t}^{0}$ of $\mathfrak{S}_{t}$ such that $\mathfrak{S}_{t}^{0} \subseteq D_{A(t)}, A(t) \mathfrak{S}_{t}^{0} \subseteq \mathfrak{S}_{t}^{0}$ and the mapping $a \rightarrow A_{0}(t)$ is a representation of the Lie algebra $(S)$ with representation space $\mathfrak{W}_{t}^{0}$.

\section{BIBLIOGRAPHY}

1. C. Chevalley, Theory of Lie groups, Princeton University Press, 1946.

2. L. Gårding, Continuous representations of Lie groups, Proc. Nat. Acad. Sci. U.S.A. vol. 33 (1947) pp. 331-332.

3. F. I. Mautner, The completeness of the irreducible unitary representations of a locally compact group, Proc. Nat. Acad. Sci. U.S.A. vol. 34 (1948) pp. 52-54.

4. - Unitary representations of locally compact groups I, Ann. of Math. vol. 51 (1950) pp. 1-25.

5. - Unitary representations of locally compact groups II, Ann. of Math. vol. 52 (1950) pp. 528-556.

6. J. v. Neumann, Zur Algebra der Funktionaloperationen und Theorie der normalen Operatoren, Math. Ann. vol. 102 (1930) pp. 197-213.

7. - On rings of operators. Reduction theory, Ann. of Math. vol. 50 (1949) pp. 401-485.

8. L. Pontrjagin, Topological groups, Princeton University Press, 1939.

Point Shirley, Mass. 